\title{
D oença arterial obstrutiva periférica: que atenção temos dispensado à abordagem clínica dos pacientes?
}

\author{
Peripheral obstructive arterial disease: what kind of clinical treatment \\ are we recommending to our patients? \\ Anaí Espinelli de Souza D urazzo ${ }^{1}$, Cid José Sitrângulo Jr.2, Calogero Presti ${ }^{2}$, \\ Erasmo Simão da Silva ${ }^{3}$, N elson D e Luccia ${ }^{4}$
}

\section{Resumo}

O bjetivo: Avaliar como pacientes com doença arterial obstrutiva periférica têm sido tratados, em nosso meio, com relação aos fatores de risco e comorbidades.

M étodo: Questionário sobre pesquisa e tratamento da dislipidemia, diabetes, exercício, uso de anti-plaquetários, tabagismo e hipertensão arterial no paciente com doença arterial obstrutiva periférica foi aplicado entre os médicos presentes na reunião mensal de março de 2004 da Sociedade Brasileira de Angiologia e Cirurgia Vascular - Regional São Paulo.

Resultados: D os 102 questionários distribuídos, 75 foram respondidos (taxa de resposta de $73,5 \%$ ). Entre os consultados, $82 \%$ pesquisam rotineiramente perfil lipídico e $20 \%$ visam alvo de LD Lcolesterol abaixo de $100 \mathrm{mg} / \mathrm{dl} ; 94 \%$ realizam pesquisa para diabetes melito; 97\% recomendam exercício; 79\% prescrevem aspirina; 97\% aconselham que os pacientes parem de fumar e $60 \%$ se restringem ao aconselhamento isoladamente; $18 \%$ não realizam a medida da pressão arterial durante a consulta e 19\% visam alvo pressórico de $130 \mathrm{x}$ $80 \mathrm{mmH}$ g. Considerando todas as avaliações em conjunto - intervenção no estilo de vida, no sentido de parar de fumar, orientação de exercícios, uso de anti-plaquetários, real ização de pesquisa para diabetes melito, controle rigoroso da pressão arterial e lípides - observou-se que $7 \%$ dos entrevistados seguem todas essas recomendações como uma rotina estabelecida.

C onclusão: 0 presente estudo demonstrou que, em nosso meio, a pesquisa e 0 tratamento dos fatores de risco e comorbidades nos pacientes com doença arterial obstrutiva periférica estão sendo subrealizados.

Palavras-chave: doenças vasculares periféricas, arteriosclerose, claudicação intermitente, fatores de risco, colesterol, tabagismo, aspirina, exercício, hipertensão, diabetes melito.

\begin{abstract}
O bjective: The purpose of this survey was to evaluate how patients with peripheral obstructive arterial disease have been treated, concerning risk factors and comorbidities.

M ethod: A questionnaire was applied to all physicians attending the monthly meeting of the Brazilian Society of Angiology and Vascular Surgery - São Paulo Section. Q uestions were asked about the following major risk factors: treatment of dyslipidemia, diabetes, exercise regimens, antiplatelet therapy, smoking and arterial hypertension.

Results: 0 f the 102 questionnaires, 75 were answered (response rate of $73.5 \%$ ). Of these, $82 \%$ routinely measure cholesterol levels and $20 \%$ aim at an LD L cholesterol target below $100 \mathrm{mg} / \mathrm{dl} ; 94 \%$ perform a screening for diabetes mellitus; $97 \%$ recommend patients to an exercise program; $79 \%$ prescribe aspirin; $97 \%$ recommend patients to quit smoking and $60 \%$ only do it by counseling; $18 \%$ do not measure blood pressure and $19 \%$ have a target pressure of $130 \times 80 \mathrm{mmH}$ g. Considering the recommendations as a whole lifestyle intervention in order to quit smoking, aerobic exercise, prescription of antiplatelet therapy, screening for diabetes mellitus, rigorous control of blood pressure and lipids - it was observed that $7 \%$ of interviewees follow all of them as an established routine.

Conclusion: The present study showed that patients with peripheral arterial disease are currently undertreated with regard to the screening and treatment of risk factors and comorbidities.
\end{abstract}

Key words: peripheral arterial disease, arteriosclerosis, intermittent claudication, risk factors, cholesterol, smoking, aspirin, exercise, hypertension, diabetes mellitus.

1. Doutora.

2. Doutor. Professor, Faculdade de Medicina, Universidade de São Paulo (USP), São Paulo, SP.

3. Doutor. Professor assistente, Faculdade de Medicina, USP, São Paulo, SP

4. Professor associado, Faculdade de Medicina, USP, São Paulo, SP.

Trabalho realizado na Regional de São Paulo da Sociedade Brasileira de Angiologia e Cirurgia Vascular. Participação em Congresso: trabalho apresentado no III Encontro São Paulo de Cirurgia Vascular, em São Paulo de 01 a 02 de abril de 2005 e vencedor do Prêmio "Encontro São Paulo de Cirurgia Vascular" na qualidade de melhor tema livre apresentado.

Artigo submetido em 19.07.05, aceito em 16.08.05. 
A aterosclerose é doença sistêmica que se desenvolve ao longo de várias décadas, piorando com 0 avançar daidade. A doença arterial obstrutiva periférica(D AO P) promove limitação funcional em conseqüência da isquemia e está associada a alto risco demorbimortalidade cardiovascular. Após os 40 anos, o risco da D AOP aumenta de duas a três vezes a cada incremento de 10 anos na idade. Estima-se que a prevalência da D AO P gire em torno de 15 a $20 \%$ em pacientes acima de 65 anos. A D AOP está associada a fatores de risco (FR) como: tabagismo, diabetes melito (D M ), hipertensão arterial sistêmica (HAS) e dislipidemia, que podem levar ao desenvolvimento generalizado eprogressivo de placas ateroscleróticas. D M etabagismo são considerados os mais importantes, cada um implicando em aumento de risco relativo para 0 desenvolvimento da DAO P em três a quatro vezes ${ }^{1-5}$.

Existe elevada associação entre DAOP, doença arterial coronariana (D AC) e doença carotídea, o que predispõe esses pacientes a aumento importante do risco de desenvolvimento de infarto agudo do miocárdio (IAM ), acidentevascular cerebral (AVC) isquêmico e morte vascular. Em pacientes com DAOP, o risco relativo de mortalidade de todas as causas está aumentado em três vezes, e o de morte vascular, em seis vezes. Esse risco é aproximadamente equivalente em homens emulheres e permaneceelevado, mesmo que o paciente não apresente evidências clínicas de doença cardiovascular (DCV). N esse contexto, a D AO P é interpretada como marcador deaterosclerosegeneral izada epreditor deinfarto eAV C. 0 tratamento clínico daD AO P deve incluir medidas que aliviem os sintomas, combinadas com medidas deprevenção das complicações cardiovasculares secundárias (IAM, AVC, isquemia aguda do membro, morte de causa cardiovascular). O s eventos trombóticosagudos, causadores de isquemia, são geralmente decorrentes da rotura da placa aterosclerótica e conseqüente oclusão trombótica do vaso ${ }^{1-5}$.

$\mathrm{N}$ a claudicação intermitente, o principal tratamento sintomático inclui interrupção do tabagismo, exercícios físicos programados e medicamentos para aumentar a tolerância ao exercício e a distância percorrida. 0 objetivo da terapêutica clínica global coadjuvante, nessesubgrupo de pacientes, deveser também a prevenção de eventos isquêmicos sistêmicos secundários ${ }^{1-5}$. N 0 caso dos pacientes que desenvolvem isquemia crítica e são submetidos a tratamento cirúrgico ou endovascular, pode-se esperar, adicionalmente, com a adoção da terapêutica clínica global, o aumento da patência da restauração a médio e longo prazo. N esse mesmo contexto, deve ser considerado que a adoção de cuidados clínicos mais efetivos em relação aos FR controláveis para a aterosclerosenos pacientessubmetidosà revascularização arterial refletirá positivamente na qualidade de vida, assim como nos índices de sobrevida dessa população.

Pacientes com D AO P sem infarto do miocárdio prévio têm o mesmo risco de apresentar evento cardiovascular do que pacientes com diagnóstico prévio de infarto, mas sem D AO P ${ }^{5,6}$. Portanto, devem ser identificados como de risco el evado para 0 desenvolvimento de eventos isquêmicos coronarianos e ser tratados, mesmo sem presença documentada de cardiopatia isquêmica, de forma equivalente a pacientes com diagnóstico prévio de isquemia miocárdica ${ }^{4-9}$. As recomendações gerais do tratamento clínico coadjuvante proposto para os pacientes com DAO P estão resumidas na T abela 1.

0 tratamento clínico é benéfico, tanto em termos locaisquanto sistêmicos ${ }^{1-5,10-14}$. Porém, éreconhecido que o tratamento da doença debase, a aterosclerose, tem sido negligenciado, tanto nos cuidados crônicos ambulatoriais quanto na internação hospitalar ${ }^{5,15-26 .}$

0 objetivo primário do presente estudo foi avaliar como médicos participantes da reunião científica da Sociedade Brasileira de Angiologia e C irurgia V ascular do Estado de São Paulo têm pesquisado e tratado os pacientes portadores de D AO P, com relação à dislipidemia, diabetes, exercício, uso de anti-plaquetários, tabagismo e hipertensão arterial.

Tabela 1 - Recomendações e metas a serem atingidas nos pacientescom doençaarterial obstrutivaperiférica

Parar de fumar

Realizar exercícios aeróbicos de rotina

IM C $=20$ a $25 \mathrm{~kg} / \mathrm{m}^{2}$

U so de anti-plaquetário

Pesquisa de diabetes melito, com meta $\mathrm{G}<100 \mathrm{mg} / \mathrm{dl}$

M edida rotineira da PA, com meta PA $<130 \times 80 \mathrm{mmH} \mathrm{g}$

Controle rigoroso do lípides séricos. $M$ etas:

- LDL $<100 \mathrm{mg} / \mathrm{dl}$

- Triglicérides $<150 \mathrm{mg} / \mathrm{dl}$

- HDL >40 mg/dl (homens)

- HDL >50 mg/dl (mulheres)

IMC = índice de massa corporal; PA = pressão arterial; $G=$ glicemia. 


\section{$M$ aterial e método}

A Sociedade Brasileira de Angiologia e Cirurgia V ascular (SBACV), através da R egional de São Paulo, teve a iniciativa de desenvolver esta pesquisa de forma total mente independente de qualquer indústria farmacêutica e sem conflitos de interesse.

A pesquisa baseou-se em questionário aplicado aos médicos presentesna reunião mensal de março de 2004 daSBACV - Regional São Paulo. Solicitou-sea colaboração no preenchimento do questionário, somenteuma única vez por profissional, anonimamente, refletindo a rotina adotada com relação aos seguintes FR maiores: dislipidemia, diabetes, atividade física, uso de antiagregantes plaquetários, tabagismo e H AS.

0 questionário, apresentado naT abela 2, émodelo adaptado do utilizado pela Sociedade de Cirurgia V ascular da G rã-B retanhael rlanda para avaliar o tratamento clínico dos pacientes com D AOP naquela Socieda$\mathrm{de}^{17}$. 0 sresultados são apresentados em valoresabsolutos e porcentagem.

\section{Resultados}

D os 102 questionários distribuídos na reunião, 75 foram respondidos (taxa de resposta de $73,5 \%$ ).

Considerando o fator de risco lípides, $82 \%$ dos entrevistados pesquisam, de rotina, o perfil lipídico. $V$ intepor cento seguem a recomendação de que o valor ideal de LD L-colesterol a ser al cançado éinferior a 100 $\mathrm{mg} / \mathrm{dl}$, e triglicéride, inferior a $150 \mathrm{mg} / \mathrm{dl}$.

A maioria dos consultados (94\%) realiza, de rotina, pesquisa para DM .

N oventa e sete por cento dos entrevistados recomendam, de rotina, exercícios para os pacientes, sendo que, desses, $42 \%$ recomendam exercício supervisionado.

Com relação aosanti-agregantesplaquetários, 79\% dosmédicos consultados recomendam aspirina de rotina. A maioria recomenda doses baixas, de até $200 \mathrm{mg}$. Como segunda opção de anti-plaquetário, 53\% dos entrevistados recomendam a ticlopidina.

Q uanto ao tabagismo, 97\% dos consultados aconselham rotineiramente que os pacientes parem de fumar, sendo quea maioria (em torno de $60 \%$ ) se restringe ao aconselhamento.

Com relação à H AS, $18 \%$ dos consultados não real izam a medida de rotina; $22 \%$ iniciam tratamen- to ou rechecagem com pressão arterial $(P A)>160 x$ $100 \mathrm{mmH}$.

A maioria (92\%) considera acompanhamento conjunto com médico da área clínica para os casos mais complexos. Q uase metade (47\%) dos cirurgiões entrevistados tem médico da área clínica em sua equipe, e menos da metade (43\%) dispõe de protocolos de tratamento clínico disponível em seu serviço.

C onsiderando to das as recomen dações em conjunto, ou seja, intervenção no estilo de vida, no sentido de parar defumar, orientação deexercíciosaeróbicos, antiagregante plaquetário, realização depesquisa para $D M$, controle rigoroso da PA, com alvo inferior a $130 \mathrm{x}$ $85 \mathrm{mmH} \mathrm{g}$, e dos lípides, com LDL alvo inferior a $100 \mathrm{mg} / \mathrm{dl}$, etriglicéridesinferior a $150 \mathrm{mg} / \mathrm{dl}$, observamos que $7 \%$ dos entrevistados seguem todas essas recomendações como uma rotina estabelecida.

A T abela 3 apresenta, deforma resumida, os resultados obtidos.

\section{D iscussão}

Pacientes com DAOP têm o mesmo alto risco de desenvolver ou morrer deD AC do queossobreviventes de um primeiro infarto ${ }^{5,6}$. Existe evidência de que 0 tratamento clínico para o controledosFR ecomorbidades nos pacientes ateroscleróticos proporciona prevenção secundária efetiva, modificando a progressão da doença aterosclerótica nas grandes artérias. As evidências na D AO P têm sido extrapoladas, em sua maioria, degrandes estudos de prevenção secundária em pacientescom D AC edeanál ise desubgrupo depacientescom doença arterial periférica associada. 0 principal mecanismo protetor éo da estabilização das placas ateroscleróticas, que reduz o risco de complicações trombóticas agudas ${ }^{1-5,11-14}$. Portanto, além do tratamento específico da doençaarterial do membro inferior, o médico que trata esses pacientes também deve dispensar atenção para o controle dos FR e ter interesse na avaliação de doença arterial em outros locais, que pode ser grave, embora assintomática. Estudos demonstram que muitos pacientes com D AOP não têm sido adequadamente pesquisados e tratados com relação aos FR e comorbidades, tanto noscuidadoscrônicosambulatoriaisquanto na internação hospitalar 5,14-26.

N o presenteestudo, $82 \%$ dos entrevistadospesquisam de rotina o perfil lipídico. Apenas a minoria dos entrevistados (20\%) busca atingir LDL inferior a 100 mg/dl. A elevação do colesterol total edesua fração 
T abela 2 - M odelo do questionário aplicado (adaptado ao utilizado pela Sociedade de Cirurgia V ascular da G rã-Bretanha e Irlanda)

\section{Lípides}

Se o paciente não tem dosagem prévia do colesterol total e frações e triglicérides, você solicita, de rotina, essas dosagens?

$\square$ N ão $\square \operatorname{Sim}$

Você inicia o tratamento a partir de qual valor de LD L?

$\square>100 \mathrm{mg} / \mathrm{dl} \quad \square>130 \mathrm{mg} / \mathrm{dl}$

Encaminho para tratamento

Q ual é o tratamento inicial que você recomenda?
$\square$ Dieta
D ieta + hipolipemiantes
H ipolipemiantes isoladamente

V ocê inicia o tratamento a partir de qual valor de triglicérides?

$\square>150 \mathrm{mg} / \mathrm{dl} \quad \square>200 \mathrm{mg} / \mathrm{dl}$

\section{D iabetes melito}

Se o paciente não sabe se é diabético, você solicita, de rotina, essa dosagem?

$\square \mathrm{N}$ ão $\square \mathrm{Sim}$

\section{Exercício}

Você orienta, de rotina, exercício aos pacientes?
$\square$ Não
$\square$ Sim, supervisionado
Sim, não supervisionado

\section{T ratamento anti-plaquetário}

Você recomenda aspirina de rotina?

$\square$ N ão $\square$ Sim

Em qual dose?
$\square 75 \mathrm{mg}$
$\square 100 \mathrm{mg}$
$200 \mathrm{mg}$
$\square 300 \mathrm{mg}$
outra dosagem:

Se a aspirina for contra-indicada ou não tolerada, qual anti-plaquetário você recomenda?
$\square$ N enhum
$\square$ Ticlopidina
$\square$ Clopidogrel
$\square$ Triflusal

\section{T abagismo}

Você rotineiramente aconselha que os pacientes parem de fumar?

$\square$ Não $\square$ Sim

Você orienta al gum tratamento específico?
$\square$ Não
Chicletes
$\square$ Emplastros
Anti-depressivos
Encaminho parauma clínica especializada

\section{H ipertensão}

Você rotineiramente mede a pressão arterial em seus pacientes?

$\square$ Não $\square \operatorname{Sim}$

A partir de qual valor da pressão arterial você encaminha para rechecagem e tratamento?
$\square>135 \times 85 \mathrm{mmHg}$
$\square>140 \times 90 \mathrm{mmH} \mathrm{g}$
$\square>160 \times 100 \mathrm{mmHg}$

\section{Controle dos fatores de risco}

N os casos complexos, você considera acompanhamento conjunto com um médico clínico?

$\square$ N ão $\square \operatorname{Sim}$

Você tem algum médico da área clínica na sua equipe?

$\square \mathrm{N}$ ão $\square \operatorname{Sim}$

Você tem algum protocolo de abordagem clínica da doença arterial obstrutiva periférica disponível em seu serviço?

$\square \mathrm{N}$ ão $\square \operatorname{Sim}$ 
LDL são FR estabelecidos para o desenvolvimento e progressão da DAOP. Em sua metanálise Cochrane avaliando terapia com estatinas em pacientes com D AO P, concluiu quea redução do colesterol provavelmente reduz a mortal idade, e existe evidência de que a estatina pode alterar a evolução clínica da D AO P27. A análise de subgrupo do estudo Scandinavian Simvastatin Survival Study G roup (4S) mostrou quea incidência de desenvolvimento ou piora da claudicação intermitente foi significativamente mais baixa em pacientes com D AC tratados com estatina ${ }^{28}$. 0 efeito do tratamento com estatinas em pacientes com DAOP foi, também, recentemente avaliado em análise de subgrupo do estudo M RC H eart Protection Study (H PS). 0 $\mathrm{HPS}^{29}$, estudo randomizado, duplo-cego e placebo controlado, avaliou o efeito sobre a mortalidade e

T abela 3 - Porcentagem de respostas positivas dos entrevistados para as condutas dos fatores pesquisados

\begin{tabular}{|c|c|c|}
\hline Fatores pesquisados & Q uestionamento de condutas & $\begin{array}{l}\% \text { de respostas } \\
\text { positivas }\end{array}$ \\
\hline Dislipidemia & $\begin{array}{l}\text { D osagem rotineira dos lípides séricos } \\
\text { Início de tratamento com } L D L>100 \mathrm{mg} / \mathrm{dl} \\
\text { Início de tratamento com } \mathrm{T}>150 \mathrm{mg} / \mathrm{dl}\end{array}$ & $\begin{array}{l}82 \% \\
20 \% \\
20 \%\end{array}$ \\
\hline Diabetes melito & D osagem rotineira da glicemia de jejum & $94 \%$ \\
\hline Atividade física & $\begin{array}{l}\text { Recomendação de exercício de rotina } \\
\text { Recomendação de exercício de rotina supervisionado } \\
\text { Recomendação de exercício de rotina não supervisionado }\end{array}$ & $\begin{array}{l}97 \% \\
42 \% \\
55 \%\end{array}$ \\
\hline Anti-agregante plaquetário & $\begin{array}{l}\text { Prescrição rotineira de aspirina } \\
\text { D ose prescrita de aspirina de até } 200 \text { mg } \\
\text { Segunda opção de anti-plaquetário: } \\
\text { N enhuma } \\
\text { Ticlopidina } \\
\text { Clopidogrel } \\
\text { O utros }\end{array}$ & $\begin{array}{r}79 \% \\
93 \% \\
\\
\\
8 \% \\
53 \% \\
27 \% \\
12 \%\end{array}$ \\
\hline Tabagismo & $\begin{array}{l}\text { A consel hamento isoladamente } \\
\text { Encaminhamento para clínica especializada } \\
\text { Prescriçãa de repositores de nicotina } \\
\text { Prescrição de anti-depressivos } \\
\text { Prescrição associada de repositores e anti-depressivos }\end{array}$ & $\begin{array}{l}57 \% \\
21 \% \\
6 \% \\
6 \% \\
7 \%\end{array}$ \\
\hline H ipertensão & $\begin{array}{l}\text { M edida rotineira da pressão arterial } \\
\text { Valor de início de tratamento: } \\
\qquad \begin{array}{l}>135 \times 85 \mathrm{mmH} \mathrm{g} \\
>140 \times 90 \mathrm{mmH} \mathrm{g} \\
\quad>160 \times 100 \mathrm{mmH} \mathrm{g}\end{array}\end{array}$ & $\begin{array}{l}82 \% \\
19 \% \\
59 \% \\
22 \%\end{array}$ \\
\hline Acompanhamento conjunto & $\begin{array}{l}\text { Considera acompanhamento conjunto nos } \\
\text { casos mais complexos }\end{array}$ & $92 \%$ \\
\hline M édico da área clínica & Presente na equipe & $47 \%$ \\
\hline Protocolos de tratamento clínico & Disponíveis no serviço & $43 \%$ \\
\hline
\end{tabular}


eventos cardiovasculares da estatina sinvastatina (40 mg/dl) em mais de 20500 pacientes portadores de aterosclerose (incluindo portadores de DAC, DAOP, D CV , D M ), osquaisforam acompanhadospor 5 anos. 0 uso da estatina sinvastatina foi associado a uma redução de $12 \%$ na taxa de mortalidade total, $17 \%$ na taxa de mortalidade vascular, $24 \%$ na incidência de eventos coronarianos, $27 \%$ na incidência de AVC e a uma redução de $16 \%$ nasrevascularizaçõesnão-coronarianas. Resultadossimilaresforam obtidosno subgrupo de 6.748 portadores de DAOP, independente dos níveis séricos basais do colesterol-LD L ${ }^{29}$. Em recente trabal ho desenvolvido em nosso meio, demonstrou-se efeito benéfico cardioprotetor do uso perioperatório da estatina atorvastatina na diminuição de eventos isquêmicos sistêmicos (IAM, AVC, angina instável e morte de causa cardíaca) nos 6 meses após cirurgia vascular arterial, quando comparada com placebo. Essa proteção foi observadaindependentementedosníveis séricos basais do colesterol ${ }^{30}$. A primeira prioridade da terapêutica farmacológica, como indicam os consensos, para o grupo de alto risco no qual os pacientes com D AO P estão incluídos, éatingir 0 al vo de LD L inferior a $100 \mathrm{mg} / \mathrm{dl}$. Estudos recentes nesse grupo demonstram queumaterapêuticamaisagressivacom al tas doses de estatina para alcançar um LD L $<70 \mathrm{mg} /$ dl proporcionam uma redução significativa nos eventos futuros. 0 risco dos efeitos colaterais com al tas doses deestatina é claramente suplantado pelo benefício que essa terapêutica oferecepara os pacientes deal to risco. Portanto, uma redução do LD L, com alvo inferior a $70 \mathrm{mg} / \mathrm{dl}$, é uma opção terapêutica em pacientes de alto risco por $D C V$. Recomenda-se que 0 triglicéride alvo deva ser $<150 \mathrm{mg} / \mathrm{dl}$ eH D L > $40 \mathrm{mg} / \mathrm{dl}$, em homens, esuperior a $50 \mathrm{mg} / \mathrm{dl}$, em mulheres ${ }^{7-10}$.

As recomendações atuais definem que o DM deve ser pesquisado em todos os pacientes com D AOP. N a presente pesquisa, a maioria dos consultados (94\%) realiza pesquisa de rotina para D M . N o estudo da G rãB retanha e Irlanda, 34\% dos entrevistados não fazem essa pesquisa ${ }^{17}$. N o estudo de Framingham, a taxa de risco de desenvolvimento de eventosisquêmicosajustada para idadeem pacientes diabéticos, comparada com não-diabéticos, foi cinco vezes maior para homens e quatro vezes maior para mulheres ${ }^{31}$. A associação de D M eD AO P tem sido avaliada eestabelecida em vários estudos. A DAOP em pacientes diabéticos é mais agressiva, com envolvimento precoce de grandes vasos. D euma maneirageral, a claudicação intermitentepare- ce ser duas vezes mais comum entre os pacientes diabé ticos do que entre os não-diabéticos. Estudos demonstram quepacientescom D M eclaudicação intermitente têm risco de 35\% de desenvolver isquemia aguda e de $21 \%$ para amputação, comparado com 19 e $3 \%$, respectivamente, entreospacientesnão-diabéticos. A progressão de isquemia crítica de membros inferiores para gangrena gira em torno de $9 \%$ nos pacientes nãodiabéticos, comparada com $40 \%$ nos diabéticos, os quais, em geral, sofrem a amputação em idade mais precoce. As amputações são 10 vezes mais freqüentes em diabéticos com doença arterial periférica do queem não-diabéticos com D AO P. A pesar dos pacientes diabéticos representarem em torno de $20 \%$ da população com DAOP, estima-se que 40 a $45 \%$ de todos os amputados de membro inferior sejam diabéticos. A fal hano diagnóstico da D M écomum eocorreem mais do que $12 \%$ decasosnovos declaudicação intermitente atendidos ambulatorial mente ${ }^{4,24,32-33}$.

0 exercício regular de moderada intensidade auxilia na manutenção do peso a longo prazo. Recomendase a manutenção do índice de massa corporal (IMC), definido pelo peso $(\mathrm{kg})$ dividido pelaaltura $\left(\mathrm{m}^{2}\right)$, entre 20 a $25 \mathrm{~kg} / \mathrm{m}^{2}$. 0 sobrepeso (I M C 25,0-29,9 kg/m²) e aobesidade (IM C $\geq 30 \mathrm{~kg} / \mathrm{m}^{2}$ ) estão claramenterelacionados com a aterosclerose eelevação do risco cardiovascular, sendo fator de risco independente para desenvolvimento de HAS, dislipidemia e doença coronariana. Além disso, o exercício regular mel hora a sensibilidade àinsulina eo controleglicêmico nosdiabéticos, além de facilitar o controle de alguns FR para DCV, como a H AS e a dislipidemia. M etanálises recentes demonstram que a distância máxima percorrida e a distância livre de dor são aumentadas pel o exercício em pacientes com claudicação intermitente, e o exercício também auxilia na tentativa de parar de fumar ${ }^{1-5}$. Em nosso meio, estudo prospectivo com 500 pacientes portadoresdeclaudicação intermitentesubmetidos atratamento clínico mostrou que aqueles que aderiram à programação de caminhadas propostas, em um período de 6 meses, obtiveram um acréscimo médio de 33 a $42 \mathrm{~m}$ por mês em seu desempenho. O s que não aderiram às caminhadas nesse período, ou não obtiveram qualquer melhora, ou obtiveram melhora desprezível em seu desempenho (média de $7 \mathrm{~m}$ por mês) ${ }^{34}$. $\mathrm{N}$ a presente pesquisa, $97 \%$ dos entrevistados recomendam, de rotina, que os pacientes adotem programa de exercícios; $42 \%$ orientam exercícios supervisionados e $55 \%$ não supervisionados. 
A recomendação genérica de exercício deve ser revista para pacientes com lesões nos pés, neuropáticas ou isquêmicas. N essascondições, caminhadas, por exemplo, podem ser prejudiciais, agravando ulcerações. Os exercícios devem ser adaptados para essas situações, ou então serem recomendados após cicatrização.

Setenta e nove por cento dos médicos consultados recomendam aspirina de rotina. Essenúmero éconcordante com pesquisasnorte-americanas, em queo uso de anti-agregantes plaquetários nos pacientes com D AOP ocorre em torno de $73 \% 24$, mas difere da pesquisa da G rã-Bretanha e I rlanda, na qual a recomendação de aspirina fica em torno de $99 \%{ }^{17}$. N o presentetrabal ho, a maioria dos médicos recomenda doses baixas, de até 200 mg, quetêm efeito protetor adequado, com menor risco de sangramento. Como segunda opção de antiplaquetário, 53\% dos entrevistados recomendam a ticlopidina. 0 uso crônico da ticlopidina tem sido limitado por seus efeitos colaterais, como aneutropenia severaeatrombocitopenia. 0 clopidogrel, escolhido na pesquisa da Grã-Bretanha e Irlanda como segunda opção de anti-plaquetário, tem eficácia comparada à ticlopidina, porém com menos efeitos colaterai ${ }^{17,35-38}$. A aspirina tem sido recomendada como terapêutica primária e secundária para prevenir eventos cardiovasculares. A contribuição dos estudos, em especial do grupo Antithrombotic T rialists' Collaboration, demonstra que, a menos que contra-indicado, todos os pacientes com doença arterial periférica se beneficiam do uso de um agente anti-agregante plaquetário. Isso porque proporcionam redução de eventos isquêmicos sistêmi$\cos ($ I AM , AVC, angina emortevascular), melhora dos sintomas da claudicação, diminuição da progressão da D AO P, danecessidade de revascularização, assim como podem, eventual mente, aumentar a patência dosenxertos vasculares. A dosagem de aspirina, na maioria dos estudos, variou entre 75 e 325 mg/d. N ão há evidências para se orientar uma dose específica, mas, utilizando a menor dosagem possível, pode-se reduzir os efeitos colaterais. H oje, por razões de custo e segurança, a aspirina (75-100 mg) é o agente de primeira escolha, não havendo evidência clara de que doses mais elevadas sejam maisefetivas, embora causem mais eventosadversos. Pacientes que não podem usar aspirina devem ser considerados para outro anti-plaquetário ${ }^{35-38}$. 0 clopidogrel também tem se mostrado eficaz em reduzir a taxa de eventos cardiovasculares nos pacientes com $D A O P$, podendo ser indicado nos pacientes que tem intolerância à aspirina ${ }^{39}$.
Com relação ao tabagismo, na presente pesquisa, $97 \%$ dos consultados aconsel ham rotineiramente que os pacientes parem de fumar, sendo que a maioria (em torno de $60 \%$ ) se restringe ao aconselhamento. 0 hábito de fumar contribui para uma em cada cinco mortes nos Estados U nidos eéa principal causa modificável de morte prematura. Existem fortes evidências de que o tabagismo é o fator de risco isolado mais importanteno desenvolvimento eprogressão daD AOP. Entre os pacientes com claudicação intermitente, 0 tabagismo está claramente associado ao desenvolvimento de claudicação limitante, isquemia crítica do membro, amputação e necessidade de intervenção cirúrgica4,10,14,40-41. Além disso, o tabagismo aumenta de forma significativa o risco de insucesso dasintervenções arteriais periféricas e morbimortalidade ${ }^{14,40-41}$. 0 diagnóstico deD AO P tendea ser feito com uma década de antecedência entre os fumantes do queentre os nãofumantes. 0 aumento de risco de câncer e dos efeitos adversos na função pulmonar persistem por muitos anos apósa interrupção do tabagismo, mas o excesso de risco deDCV (morte eIAM não-fatal) diminui relativamente rápido - em 2 a 4 anos - após a cessação do tabagismo. A despeito do claro benefício da cessação de tabagismo, somente uma minoria de pacientes al cança esseobjetivo. A pesar de 70\% dostabagistasexpressarem desejo de parar de fumar, menos de um terço deles consegue, e somente $5 \%$ mantêm-se sem fumar a cada ano; a maioria, após várias falhas, abandona as tentativas. Baseado em outras pesquisas, sabe-sequeo aconseIhamento, isoladamente, produz modestas taxas de sucesso. Revisão de 108 estudos randomizados controlados demonstrou que os repositores de nicotina são efetivos como parte da estratégia de parar de fumar e aumenta a taxa de sucesso na interrupção do tabagismo em 1,5 a 2 vezes. 0 efeito dos repositores de nicotina é independente de qualquer outro suporte adicional $14,41,42$.

C om relação à H AS, na presente pesquisa, 18\% dos consultados não realizam a medida da PA de rotina. Pesquisa norte-americana, avaliando o tratamento clínico dosFR ecomorbidades nospacientescom DAOP, demonstra que a PA foi medida em todos os pacientes internados para procedimento cirúrgico vascular, mas em menosde $50 \%$ dospacientesambulatoriais ${ }^{24}$. Vinte e sete por cento dos entrevistados na pesquisa da GrãBretanha e I rlanda não realizam a medida pressórica de rotina, e $41 \%$ iniciam tratamento ou rechecagem com $\mathrm{PA}>160 \times 100 \mathrm{mmH} \mathrm{g}^{17}$. N a presente pesquisa, em 
torno de $80 \%$ dos médicos encaminham os pacientes para rechecagem ou início de tratamento com níveis pressóricos mais baixos do que $160 \times 100 \mathrm{mmH} \mathrm{g}$. A H AS estáassociadaa um aumento do risco de desenvolvimento de D AOP em duas a três vezes. A definição de hipertensão na população geral corresponde a níveis de PA sistólica maior que $140 \mathrm{mmH}$ g, ou diastólica maior que $90 \mathrm{mmH}$ g. No entanto, esses níveis são considerados excessivos para os pacientes com D AOP. Estudos epidemiológicos e ensaios clínicos randomizados demonstram haver benefício contínuo na diminuição de eventos cardiovasculares pela redução dos níveis tensionais para val ores menores do que $130 \times 80 \mathrm{mmH}$ g. As recomendações atuais sugerem a instituição de tratamento agressivo da PA nos pacientes portadores de aterosclerose, orientando que os pacientes com D AOP estão incluídosnesse subgrupo dealto risco. 0 objetivo do tratamento seria obter uma PA abaixo de $130 \mathrm{x}$ $80 \mathrm{mmH}$ g. Apesar dos ß-bloqueadores já terem sido considerados contra-indicados nos pacientes com DAOP, pela possibilidade da piora dos sintomas da doença, essa preocupação não foi confirmada em estudos randomizados, podendo, portanto, ser usados nesses pacientes. Aliás, já que os pacientescom D AOP têm elevada incidência de D AC concomitante, o uso de ßbloqueadores oferece o benefício adicional da cardioproteção ${ }^{1-5,13,43}$. O s inibidores da enzima de conversão da angiotensina (ECA) têm se mostrado benéficos no controle pressórico e na redução de eventos cardiovasculares nos pacientes de alto risco, entre eles os portadoresdeD AOP, sejam portadoresou não deD M , como demonstrado pelo estudo $\mathrm{H}$ eart O utcomes Pre vention Evaluation (HOPE) ${ }^{13}$.

Esta pesquisa demonstrou falta de diretrizes locais. $M$ enos da metade (43\%) dos entrevistados dispõe de protocolos de tratamento clínico em seu serviço. Entretanto, evidenciou que a maioria dos cirurgiões vasculares considera importante 0 acompanhamento conjunto com médicos da área clínica nos casos mais complicados. $\mathrm{N}$ a prática, 0 acompanhamento conjunto é disponível em $47 \%$ das equipes de cirurgiões vasculares.

Considerando todas as recomendações em conjunto, ou seja, intervenção no estilo de vida, no sentido de parar de fumar, orientação de exercícios aeróbicos, anti-plaquetário, realização de pesquisa para D M , controlerigoroso da PA, com alvo inferior a $130 \times 80 \mathrm{mmH} \mathrm{g}$, e dos lípides, com LDL alvo inferior a $100 \mathrm{mg} / \mathrm{dl}$, observamos que apenas uma pequena porcentagem ( $7 \%$ ) dos entrevistados segue essas recomendações como uma rotina estabel ecida. A mai oria dos pacientes com $D$ AO P tem mais do que um FR modificável; a associação de FR tem mais do que um efeito aditivo sobre o risco total ${ }^{31}$. 0 resultado da pesquisa da G rã-Bretanha e Irlanda - à semelhança de outras pesquisas norte-americanas e européias - demonstrou que o tratamento clínico foi sub-utilizado ${ }^{5,15-26}$. 0 presente estudo demonstrou que, também em nosso meio, a pesquisa e o tratamento dos FR e comorbidades estão sendo subrealizados.

C onsiderando dados da literatura sobrea eficácia do tratamento clínico globalizado da doença aterosclerótica em relação aos FR, e o baixo engajamento dos médicos entrevistados na prescrição dessa terapêutica aos pacientes arteriopatas periféricos, como demonstrado nesta pesquisa, torna-se um desafio para a SBACV propor diretrizes, baseadas em evidências, para o tratamento global do paciente portador de doença arterial obstrutiva periférica.

\section{Agradecimentos}

O sautores agradecem aos colegas da Regional de São Paulo da Sociedade Brasileira de Angiologia e Cirurgia $V$ ascular que colaboraram com o preenchimento dos questionários, permitindo a realização desta pesquisa.

\section{R eferências}

1. Burns P, LimaE, Bradbury W. W hat constitutes best medical therapy for peripheral arterial disease? Eur J V asc Endovasc Surg. 2002;24:1-12.

2. H iatt WR. Pharmacologic therapy for peripheral arterial disease and claudication. J V asc Surg. 2002;36:1283-91.

3. O uriel K. Peripheral arterial disease. Lancet. 2001;358: 1257-64.

4. Task W orking G roup. M anagement of Peripheral Arterial D isease (PAD) TransAtlantic Inter-Society Consensus (TASC). J V asc Surg. 2000;31(1 Pt 2):3-9.

5. Rehring TF, Sandhoff BG, Stolcpart RS, M erenich JA, H ollis $\mathrm{HW}$. Atherosclerotic risk factor control in patients with peripheral arterial disease. J Vasc Surg. 2005;41:816-22.

6. $H$ affner $M$, Lehto $S$, Ronnemaa $T$, Pyorala $K$, Laakso M . $M$ ortality from coronary heart disease in subjects with type 2 diabetes and in nondiabetic subjects with and without prior myocardial infarction. N EngJ Med. 1998;339:229-34. 
7. Grundy SM, Cleeman JI, M erz N B, et al. Implications of recent clinical trials for the $\mathrm{N}$ ational $C$ holesterol Education Program Adult Treatment Panel III Guidelines. J Am Coll Cardiol. 2004;44:720-32.

8. Joint British recommendations on prevention of coronary heart disease in clinical practice. British Cardiac Society, British $\mathrm{H}$ yperlipidaemia Association, British $\mathrm{H}$ ypertension Society, endorsed by the British D iabetic Association. H eart. 1998;80:S1-29.

9. III D iretrizes Brasileiras Sobre Dislipidemias e Diretriz de Preven ção daA terosclerose do D epartamento deA terosclerose da Sociedade Brasileira de Cardiologia. Arq Bras Cardiol. 2001;77:4-48.

10. $H$ irsch AT, T reat-Jacobson D, Lando H A, H atsukamo DK. The role of tobacco cessation, antiplatelet and anti lipidlowering therapies in the treatment of peripheral arterial disease. J V asc M ed. 1996;2:243-51.

11. Schwartz GG, O Isson AG, Ezekowitz M D, Ganz P, O liver $M F, W$ aters D. M yocardial I schemiaR eduction with Aggressive C holesterol Lowering(M IRACLE) Study I nvestigators. Effects of atorvastatin on early recurrent ischemic events en coronary syndromes: a randomized controlled trial. JAM A. 2001;285:1758-60.

12. Burns $P, G$ ough S, Bradbury AW . M anagement of peripheral arterial disease in primary care. BM J . 2003;326:584-8.

13. The $\mathrm{H}$ eart Outcomes Prevention Evaluation Study Investigators. Effects of an angiotensin-converting enzyme inhibitor, ramipril, on cardiovascular events in high-risk patients. N Engl J Med. 2000;342:145-53.

14. Donnelly $R$, Yeung JM. M anagement of intermittent claudication: the importance of secondary prevention. Eur J V asc Endovasc Surg. 2002;23:100-7.

15. H enkePK, Blackburn S, Proctor M C, et al. Patientsundergoing infrainguinal bypass to treat atherosclerotic vascular disease are underprescribed cardioprotective medications: Effect on graft patency, limb salvage, and mortality. J Vasc Surg. 2004;39:357-65.

16. M ukherjee $D$, Lingham $P$, Chetcuti $S, G$ rossman $M$, et al. $M$ issed opportunities to treat atherosclerosis in patients undergoing peripheral vascular interventions. Circulation. 2002;106:1909-12.

17. C assarK, Belch JJ, Brittenden J. A renational cardiac guidelines being applied by vascular surgeons? Eur J V asc Endovasc Surg. 2003;26:623-8.

18. Clark AL, Byrne JC, N asser A, M CG roarty E, Kennedy JA. Cholesterol in peripheral vascular disease- a suitable case for treatment? Q JM . 1999;92:219-22.

19. Bismuth J, Klitfod L, Sillesen H. The lack of cardiovascular risk factor management in patientswith critical limb ischaemia. Eur J V asc Endovasc Surg. 2001;21:143-6.

20. Anand SS, Kundi A, Eikelboom J, Yusuf S. Low rates of preventivepracticesin patients with peripheral vascular disease. Can J Cardiol. 1999;15:1259-63.

21. M CD ermott M M, M ehta S, Ahn H, Greenland $P$. Atherosclerotic risk factors are less intensively treated in patients with peripheral arterial disease than in patients with coronary artery disease. J G en Intern M ed. 1997;12:209-15.
22. $M$ CD ermott M M , H ahn EA, G reenland $P$, et al. Atherosclerotic risk factor reduction in peripheral arterial disease: results of a national physician survey. J Gen Intern Med. 2002;17: 895-904.

23. H irsch AT, G otto AM Jr. U ndertreatment of dyslipidemia in peripheral arterial disease and other high-risk populations: an opportunity for cardiovascular disease reduction. V asc $M$ ed. 2002;7:323-31.

24. BurnsP, LimaE, Bradbury AW. Second best medical therapy. Eur J Vasc Endovasc Surg. 2002;24:400-4.

25. $M$ ukherjeeD, Lingan $P, C$ hetcuti $S, G$ rossman $P M, M$ oscucci $M$, Luciano $A E$, Eagle K. M issed opportunities to treat atherosclerosis in patients undergoing peripheral vascular interventions: insights from the U niversity of $M$ ichigan Peripheral vascular disease quality improvement initiative (PVD-QI). Circulation. 2002;106:1909-12.

26. N aylor AR. Does the modern concept of "best medical therapy" render carotid surgery obsolete?E ur J V asc Endovasc Surg. 2004;28:457-61.

27. Leng GC, Price JF, Jepson RG. Cochrane review: lipidlowering therapy in thetreatment of lower limb atherosclerosis. Eur J V asc Endovasc Surg. 1998;16:5-6.

28. Pederson TR, Kjekshus], PyoralaK, et al. Effect of simvastatin on ischaemic signs and symptoms in the Scandinavian Simvastatin Survival (4S) study. Am J Cardiol. 1998;81:333-5.

29. Heart Protection Study Collaborative Group. M RC/BHF $\mathrm{H}$ eart Protection Study of cholesterol lowering with simvastatin in 20,536 high-risk individuals: a randomised placebocontrolled trial. Lancet. 2002;360:7-22.

30. D urazzo AE, M achado FS, Ikeoka DT, et al. Reduction of cardiovascular events after vascular surgery with atorvastatin: a randomized trial. J V asc Surg. 2004;39:967-76.

31. M urabito JM, D 'Agostinho RB, Silbershatz H, W ilson PW . Intermittent claudication: a risk profilefrom theFramingham H eart Study. Circulation. 1997;96:44-9.

32. D e Luccia N . D oença vascular e diabetes. J V asc Br. 2003;2: 49-60.

33. $M C D$ aniel $M D, C$ ronenwett $J L$. Basic data related to the natural history of intermittent claudication. Ann Vasc Surg. 1989;3:273-7.

34. W olosker N, N akano L, R osoky RA, Puech-Leão P. Evaluation of walking capacity over timein 500 patients with intermittent claudication who underwent clinical treatment. Arch Intern M ed. 2003;163:2296-300.

35. Peripheral Arterial Disease Antiplatelet Consensus Group. Antiplatelet Therapy in Peripheral Arterial D isease. C onsensus Statement. Eur J V asc Endovasc Surg. 2003;26:1-16.

36. Antiplatelet Trialists'C ollaboration. Collaborative overview of randomized trials of antiplatelet therapy. I: Prevention of death, myocardial infarction, and stroke by prolonged antiplatelet therapy in various categories of patients. BM J. 1994;308:81-106.

37. Robless P, M ikhailidis D P, Stansby G. Systematic review of antiplatel et therapy for theprevention of myocardial infarction, stroke and vascular death in patients with peripheral arterial disease. Br J Surg. 2001;88:787-800. 
38. Antiplatelet Trialists'C ollaboration. Collaborative overview of randomized trials of antiplatelet therapy. II: M aintenance of vascular graft or arterial patency by antiplatelet therapy. BM J. 1994;308:159-68.

39. CAPRIE Steering C ommittee. A randomized, blinded trial of clopidogrel versusaspirin in patients at risk of ischemic events (CAPRIE). Lancet. 1996;348:1329-39.

40. Ameli FM, Stein M, Provan JL, Prosser R. The effect of postoperative smoking on femoropopliteal bypass graft. Ann V asc Surg. 1989;3:20-25.

41. M inistério daSaúde, Instituto $\mathrm{N}$ acional do $\mathrm{C}$ ancer. A bordagem e T ratamento do Fumante - Consenso 2001. Rio de Janeiro: IN CA, 2001. 38p.

42. Hobbs SD, Bradbury AW. Smoking cessation strategies in patients with peripheral arterial disease: an evidence-based approach. Eur J V asc Endovasc Surg. 2003;26:341-7.
43. G rupo detrabalho paralV D iretrizesBrasileirasdeH iperten são Arterial. IV D iretrizes Brasileiras deH ipertensão Arterial. Arq Bras Cardiol. 2004;82 Supl IV:7-22.

Correspondência:

Anaí Espinelli de Souza D urazzo

Rua Augusta, 1819/141

CEP 01413-000 - São Paulo, SP

Tel.: (11) 3289.2458

E-mail: anaidurazzo@uol.com.br

0 arquivo disponível sofreu correções conforme ERRATA publicada no Volume 4 Número 4. 